\title{
Vergleiche verschiedener Gesundheitswesen: Parallelen und Überraschungen
}

\author{
Zur Beurteilung eines Gesundheitssystems sind internationale Vergleiche unent- \\ behrlich. Die Organisation für wirtschaftliche Zusammenarbeit und Entwicklung \\ OECD hat neben der WHO die grössten Datenbanken. Die Analyse ergibt: Vergleich- \\ bare Länder zahlen im Durchschnitt $24 \%$ weniger für ihr Gesundheitswesen als die \\ Schweiz.
}

Korrespondenz:

Dr. rer. pol. Gerhard Kocher Haldenweg $10 \mathrm{~A}$ CH-3074 Muri

gerhard.kocher[at]muri-be.ch
73,1 Milliarden Franken werden wir dieses Jahr für unser Gesundheitswesen ausgeben. Diese Schätzung beruht auf der provisorischen Zahl des Bundesamtes für Statistik [1] für 2012 (68 Milliarden Franken). Zwischen 2007 und 2012 nahmen die Ausgaben im Durchschnitt um 2,54 Milliarden pro Jahr zu. Nimmt man diesen Betrag als Schätzwert auch für 2013 und 2014, ergibt dies 73 Milliarden für 2014. Ist es nicht seltsam, dass seit Jahrzehnten in unserem Gesundheitssystem über Sparmassnahmen diskutiert und da und dort auch hart gespart wird, wir dann aber gleichwohl Jahr für Jahr zweieinhalb Milliarden Franken zusätzlich ins System buttern?

Zur Beurteilung eines Gesundheitssystems sind internationale Vergleiche unentbehrlich. Die Organisation für wirtschaftliche Zusammenarbeit und Entwicklung OECD in Paris hat dafür neben der WHO die grössten Datenbanken über die Gesundheitswesen ihrer 34 Mitgliedsländer [2]. Leider werden die OECD Health Statistics 2014 nicht mehr auf einer CD veröffentlicht, sondern nur noch in Auszügen als Exceldateien [3]. Wer ausführliche Daten braucht, hat mit einem Online-Abonnement für jährlich 326 Euro freien Zugang zur Datenbank mit allen Gesundheitsstatistiken [4]. Den grossen thematischen Umfang dieser Datenbank zeigt eine 17-seitige Liste mit allen erfassten Variablen [5].

\section{Unterschiede in der Höhe der Gesund- heitsausgaben}

2012 gaben die OECD-Länder im Schnitt pro Einwohner 3484 USD für ihr Gesundheitswesen aus (Tab. 1). Die Ausgabenunterschiede sind beträchtlich, wie ein Vergleich der Extreme zeigt: Die USA geben fast neun Mal mehr aus als die Türkei [6]. Wie seit Jahren sprengen die US-Gesundheitsausgaben alle Grenzen. Sie sind 151\% höher als der OECD-Durchschnitt, $42 \%$ höher als im zweitplatzierten Norwegen und $44 \%$ höher als in der drittplatzierten Schweiz.

Der Vergleich mit dem Sonderfall USA darf nicht darüber hinwegtäuschen, dass auch wir uns ein extrem teures Gesundheitssystem leisten. Unsere 6080USD

\section{Comparaison de différents systèmes de santé: parallèles et surprises}

En 2014, la Suisse dépensera environ 73 milliards de francs pour son système de santé. L'ardoise augmente de 2,5 milliards chaque année, malgré tous les discours sur la nécessité de faire des économies et quelques mesures drastiques prises çà et là. Selon les derniers chiffres de l'OCDE pour 2012, nous sommes en troisième position des pays de I'OCDE pour les dépenses de santé par habitant, et même en deuxième position derrière les Etats-Unis en pourcentage du produit intérieur brut. Selon les sources, 20 à 40 pour cent des sommes engagées sont mal allouées ou gaspillées, ce qui représente en Suisse un potentiel théorique d'économies de 15 à 30 milliards de francs. La densité de médecins est de 3,9 pour 1000 habitants, plus élevée que la densité moyenne de 3,2 dans les pays de l'OCDE. Nous avons la plus haute densité de personnel soignant de tous les pays $(16,6$ infirmiers/-ères pour 1000 habitants), même si des différences de définition font que ce chiffre de I'OCDE est certainement trop élevé. L'Allemagne en affiche 11,3, la moyenne des pays de l'OCDE est de 9,1 . Pour ce qui est de la satisfaction de la population par rapport à son système de santé national, nous sommes en deuxième position selon un sondage d'opinion mené dans 11 pays. La Suisse a atteint le même rang dans une évaluation de la qualité d'après 80 indicateurs de performance. Le vainqueur des deux classements est un pays qui ne dépense qu'un peu plus que la moitié de la Suisse pour son système de santé par habitant: la Grande-Bretagne. 
Tabelle 1

Gesundheitsausgaben 2012 pro Kopf (kaufkraftbereinigt) und in Prozent des Bruttoinlandprodukts (BIP).

\begin{tabular}{|c|c|c|c|c|c|}
\hline \multirow[b]{2}{*}{ Land } & \multicolumn{2}{|l|}{2012} & \multirow{2}{*}{$\begin{array}{r}\text { Zunahme } \\
2000 \text { bis } \\
2012(\%)\end{array}$} & \multicolumn{2}{|l|}{2012} \\
\hline & Pro Kopf, USD & Index * & & In \% des BIP & Index * \\
\hline USA & 8745 & 251 & 83 & 16,9 & 182 \\
\hline Norwegen & 6140 & 176 & 102 & 9,3 & 100 \\
\hline Schweiz & 6080 & 175 & 89 & 11,4 & 123 \\
\hline Niederlande & 5099 & 146 & 129 & 11,8 & 127 \\
\hline Österreich & 4896 & 141 & 69 & 11,1 & 119 \\
\hline Deutschland & 4811 & 138 & 79 & 11,3 & 122 \\
\hline Dänemark & 4698 & 135 & 87 & 11,0 & 118 \\
\hline Kanada & 4602 & 132 & 83 & 10,9 & 117 \\
\hline Luxemburg & 4578 & 131 & 40 & 7,1 & 76 \\
\hline Belgien & 4419 & 127 & 97 & 10,9 & 117 \\
\hline Frankreich & 4288 & 123 & 68 & 11,6 & 125 \\
\hline Schweden & 4106 & 118 & 79 & 9,6 & 103 \\
\hline Australien ** & 3997 & 115 & 75 & 9,1 & 98 \\
\hline Irland & 3890 & 112 & 118 & 8,9 & 96 \\
\hline Japan & 3649 & 105 & 85 & 10,3 & 111 \\
\hline Finnland & 3559 & 102 & 92 & 9,1 & 98 \\
\hline Island & 3536 & 101 & 29 & 9,0 & 97 \\
\hline Grossbritannien & 3289 & 94 & 80 & 9,3 & 100 \\
\hline Italien & 3209 & 92 & 58 & 9,2 & 99 \\
\hline Neuseeland ** & 3172 & 91 & 97 & 10,0 & 108 \\
\hline Spanien ** & 2998 & 86 & 95 & 9,4 & 101 \\
\hline Slowenien & 2667 & 77 & 84 & 9,4 & 101 \\
\hline Portugal & 2457 & 71 & 61 & 9,5 & 102 \\
\hline Griechenland & 2409 & 69 & 66 & 9,3 & 100 \\
\hline Israel & 2304 & 66 & 30 & 7,3 & 78 \\
\hline Südkorea & 2291 & 66 & 199 & 7,6 & 82 \\
\hline Slowakei & 2105 & 60 & 247 & 8,1 & 87 \\
\hline Tschechien & 2077 & 60 & 111 & 7,5 & 81 \\
\hline Ungarn & 1803 & 52 & 111 & 8,0 & 86 \\
\hline Chile & 1577 & 45 & 159 & 7,3 & 78 \\
\hline Polen & 1540 & 44 & 164 & 6,8 & 73 \\
\hline Estland & 1447 & 42 & 182 & 5,9 & 63 \\
\hline Mexiko & 1048 & 30 & 111 & 6,2 & 67 \\
\hline Türkei & 984 & 28 & 127 & 5,4 & 58 \\
\hline $\begin{array}{l}\text { Durchschnitt } \\
\text { (34 Länder) }\end{array}$ & 3484 & 100 & 100 & 9,3 & 100 \\
\hline
\end{tabular}

* Durchschnitt aller Länder $=100$

** 2011

pro Jahr liegen $75 \%$ über dem OECD-Durchschnitt. In der oberen Hälfte der Länderliste sind zahlreiche Länder aufgeführt, die für ihr sehr gutes oder gutes Gesundheitswesen pro Kopf jährlich 10-40\% weniger ausgeben als wir. Bereits für 2010 zeigte sich, dass vergleichbare Länder (Dänemark, Österreich, Deutschland, Frankreich, Belgien, Schweden und Finnland) im Durchschnitt 24\% weniger für ihr Gesundheitswesen zahlten als die Schweiz [7].
Seit 2000 verdoppelten sich die Pro-Kopf-Ausgaben (Spalte 4, Tab. 1). Überdurchschnittlich war das Wachstum in 12 Ländern. Neun davon blieben 2012 gleichwohl in den letzten Rängen des Ausgabenrankings und gaben mit 1652 USD im Durchschnitt nicht einmal halb so viel aus wie die OECD-Länder.

Die Ausgaben 2012 der 34 OECD-Länder entsprechen im Schnitt 9,3\% des Bruttoinlandprodukts (Spalten 5 und 6, Tab. 1). Nach den USA, den Niederlanden und Frankreich ist die Schweiz hier im vierten Rang mit 11,4\%. Dies ist 23\% höher als der OECDDurchschnitt. Bei diesen Vergleichen mit dem BIP hängt der Prozentsatz natürlich nicht nur von den Gesundheitsausgaben ab, sondern auch von der Wirtschaftslage. Sie unterlag gerade in den letzten Jahren grossen jährlichen Schwankungen und entwickelte sich zudem je nach Land sehr unterschiedlich. Zur Beurteilung der Gesundheitsausgaben eines Landes müssen immer beide Datenreihen herangezogen werden: Ausgaben pro Kopf (Spalte 2) und Ausgaben im Verhältnis zum BIP (Spalte 5).

Ein weiterer Punkt ist zu beachten: Eine jährliche Kostenzunahme von beispielsweise 500 USD pro Kopf und Jahr entspricht in der Schweiz einem Wachstum von $8 \%$. In einem der ärmeren Länder bedeutet die Zunahme von 500 USD ein Ausgabenwachstum von 40 oder $50 \%$. Dies würde dort zu einer erheblichen Verbesserung des Gesundheitswesens führen. Bei uns dagegen oder gar in den USA wären die Wirkungen viel geringer. Dies ist zum Teil mit dem Phänomen des abnehmenden Grenznutzens zusätzlicher Investitionen zu erklären. Es ist auf vielen Gebieten zu beobachten. Zum Beispiel leuchtet es ein, dass eine Lohnerhöhung um 1000 Franken pro Monat für einen Angestellten mit 4000 Franken Monatslohn eine viel grössere Bedeutung und auch Wirkung hat als für jemanden, der bereits 10000 Franken oder mehr verdient.

\section{Ausgabenhöhe und die Grundübel Fehlallokationen und Verschwendung}

Komplizierter und weniger beachtet ist der Umstand, dass es nicht nur auf die Ausgabenhöhe ankommt, sondern auch darauf, wie wirksam und wie effizient dieses Geld eingesetzt wird. In vielen Ländern, auch der Schweiz, ist das Gesundheitssystem ein Reich der Verschwendung. Vor allem in den USA sind dazu ungezählte Studien erschienen. Vor genau 40 Jahren berechnete Sydney Wolfe, dass ein Viertel aller damaligen Gesundheitsausgaben überflüssig waren (wie unnötige Hospitalisationen, Spitalbetten, Chirurgie, Medikamente, Röntgenaufnahmen). Auf 26\% kam eine Untersuchung der Citizen's Health Research Group von Ralph Nader.

In der Schweiz kritisierten renommierte Fachleute ebenfalls schon damals die Verschwendung: Nach dem Lausanner Statistikprofessor Pierre Gilliand (SP) könnten wir ohne Qualitätsverlust 15\% der jährlichen Gesundheitsausgaben sparen, nach dem 
Zürcher Prof. Meinrad Schär (Landesring der Unabhängigen) einen Viertel und gemäss dem Berner Gesundheitsökonom Pierre Gygi (FDP) sogar die Hälfte. 1979/80 erschien das Buch «Teure Medizin», das im Detail Kosten und Nutzen der Medizin analysierte [8].

2012 publizierte das renommierte Institute of Medicine (IOM) in Washington eine Studie [9], wonach 2009 in den USA von den nationalen Gesundheitsausgaben (2,7 Billionen USD) 810 Milliarden oder 30\% verschwendet wurden (unnötige Leistungen, Ineffizienz, Betrug und Missbrauch, Wucherpreise und zu hoher Administrativaufwand).

Der Bundesrat verabschiedete 2013 «Die gesundheitspolitischen Prioritäten des Bundesrates», Gesundheit2020. Darin heisst es: «Experten und Expertinnen gehen davon aus, dass die heutigen Leistungen ohne Qualitätseinbussen rund 20 Prozent günstiger erbracht werden könnten» [10]. Mit dem Programm 2020 sollen diese «Effizienzreserven» angegangen werden.

\section{Tabelle 2}

Selbstzahlungen der Privathaushalte, 2012, in USD (kaufkraftbereinigt) pro Person und in Prozent der nationalen Gesundheitsausgaben.

\begin{tabular}{|c|c|c|c|c|}
\hline & USD, KKB & Index * & $\begin{array}{r}\text { Prozent aller } \\
\text { Gesundheits- } \\
\text { ausgaben }\end{array}$ & Index * \\
\hline Schweiz & 1582 & 268 & 26 & 137 \\
\hline USA & 1045 & 177 & 12 & 63 \\
\hline Belgien & 901 & 153 & 20,4 & 107 \\
\hline Norwegen & 829 & 141 & 15 & 79 \\
\hline Österreich & 769 & 130 & 16,7 & 88 \\
\hline Australien & 731 & 124 & 18,3 & 96 \\
\hline Portugal & 722 & 122 & 27,3 & 144 \\
\hline Kanada & 690 & 117 & 15 & 79 \\
\hline Griechenland & 685 & 116 & 28,4 & 149 \\
\hline Finnland & 664 & 113 & 18,7 & 98 \\
\hline Irland & 657 & 111 & 16,9 & 89 \\
\hline Island & 638 & 108 & 18,1 & 95 \\
\hline Deutschland & 627 & 106 & 13 & 68 \\
\hline Italien & 603 & 102 & 18,8 & 99 \\
\hline Israel & 597 & 101 & 25,9 & 136 \\
\hline Dänemark & 584 & 99 & 12,4 & 65 \\
\hline Ungarn & 509 & 86 & 28,3 & 149 \\
\hline Luxemburg & 506 & 86 & 11 & 58 \\
\hline Japan & 483 & 82 & 14 & 74 \\
\hline Mexiko & 474 & 80 & 45,2 & 238 \\
\hline Slowakei & 471 & 80 & 22,4 & 118 \\
\hline Polen & 350 & 59 & 22,7 & 119 \\
\hline Neuseeland & 347 & 59 & 10,9 & 57 \\
\hline Slowenien & 317 & 54 & 11,9 & 63 \\
\hline Tschechien & 312 & 53 & 15 & 79 \\
\hline Türkei & 151 & 26 & 15,4 & 81 \\
\hline Durchschnitt & 590 & 100 & 19 & 100 \\
\hline
\end{tabular}

* Durchschnitt aller Länder = Index 100
Ob die überflüssigen Gesundheitsausgaben 10 oder 20 oder mehr Prozent betragen, lässt sich nicht genau eruieren. Sicher ist aber in verschiedenen Ländern wie den USA und der Schweiz das Gesundheitssystem aufgebläht und vergoldet. Und leider auch weitgehend reform- und sparresistent. Das Volk kritisiert, dass wir für das Gesundheitswesen zu viel ausgeben und fordert deshalb folgerichtig, dass wir noch mehr ausgeben ... Eine mögliche Strategieänderung könnte darin bestehen, ein gewisses Wachstum der Gesundheitsausgaben hinzunehmen, sich dafür aber darauf zu konzentrieren, dass sie mehr Wirkung und Qualität erzielen.

\section{Ausgabensteuerung über das Portemonnaie des Kranken?}

Seit jeher ist unser Land der Champion in der finanziellen Direktbelastung der Kranken, den sog. Outof-pocket-Zahlungen [11]. Auch die neuesten OECDZahlen belegen diese Besonderheit in unserem Finanzierungsmechanismus (Tab. 2). Mit nicht weniger als 1582 USD Selbstzahlungen pro Kopf und Jahr sind wir an einsamer Spitze: zweieinhalbmal höher als der OECD-Durchschnitt und 50\% mehr als die USA. So viel zahlen wir aus dem eigenen Sack vor allem für Pflegeheime, Institutionen für Behinderte, rezeptfreie Medikamente, die Zahnmedizin, Brillen und Hörgeräte sowie Franchisen und Selbstbehalte (die Versicherungsprämien sind in den Out-of-pocketZahlen nicht inbegriffen). Insgesamt sind dies 26 Prozent aller Gesundheitsausgaben des Landes, gemäss Bundesamt für Statistik 17,144 Milliarden Franken. Nur Südkorea und einige ärmere Länder belasten ihre Bevölkerung mit einem noch höheren Direktzahleranteil an den nationalen Gesundheitsausgaben. Ganz am Schluss der Rangliste der 34 OECDLänder sind die Niederlande, Frankreich und Grossbritannien, die ihre Gesundheitssysteme nur mit 6, 7,5 und 9 Prozent über Out-of-pocket-Zahlungen finanzieren.

In kaum einem anderen Land ist der Glaube in Politiker- und anderen Kreisen noch so verbreitet wie in der Schweiz, mit hoher Selbstbelastung der Kranken liesse sich der Anstieg der Gesundheitsausgaben wirksam verringern. Bei einigen begrenzten Massnahmen sind Spareffekte möglich. Das ökonomistische Menschenbild vom Homo oeconomicus hat sich aber längst als unrealistisch erwiesen, gerade bei Patienten. Gegen höhere finanzielle Belastung des Kranken als vermeintliches Allheilmittel spricht zudem eine alte Faustregel: Eine tiefe Selbstbeteiligung ist realisierbar und sozial, aber unwirksam. Eine hohe Selbstbeteiligung ist wirksam, aber unsozial und politisch nicht realisierbar.

\section{Ärzte und Pflegefachpersonen pro 1000 Einwohner}

Die Klagen über den Personalmangel könnten bald gleich heftig werden wie die Kritik an den Kosten des 
Tabelle 3

Ärzte- und Pflegepersonen-Dichte. Praktizierende Ärzte pro 1000 Einwohner; Pflegefachpersonen pro 1000 Einwohner, die direkt in der Pflege tätig sind.

\begin{tabular}{|c|c|c|c|c|c|c|}
\hline & $\begin{array}{r}\text { Ärzte } \\
2000\end{array}$ & 2012 & $\begin{array}{r}\text { Zu-/Abnahme } \\
2000-2012 \\
\text { (in \%) }\end{array}$ & 2000 & \multicolumn{2}{|c|}{$\begin{array}{r}\text { Pflegefachpersonen } \\
2012 \text { Zu-/Abnahme } \\
2000-2012 \\
\text { (in \%) }\end{array}$} \\
\hline Australien & 2,5 & 3,3 & 32 & 10,1 & 10,2 & 1 \\
\hline Belgien & 2,8 & 2,9 & 4 & & 9,5 & \\
\hline Deutschland & 3,3 & 4 & 21 & 9,6 & 11,3 & 18 \\
\hline Estland & 3,1 & 3,3 & 6 & 5,8 & 6,2 & 7 \\
\hline Finnland (1) & 2,9 & 3,3 & 14 & 9,3 & 10,5 & 13 \\
\hline Frankreich (1) (2) & 3,3 & 3,3 & 0 & 79 & & \\
\hline Griechenland (1) (2) & 4,3 & 6,2 & 44 & 88 & & \\
\hline Grossbritannien & 2 & 2,8 & 40 & 9,0 & 8,2 & -9 \\
\hline Island & 3,4 & 3,6 & 6 & 13,3 & 15,2 & 14 \\
\hline Israel & 3,5 & 3,3 & -6 & 5,4 & 4,8 & -11 \\
\hline Italien (2) & & 3,9 & & & & \\
\hline Japan & 1,9 & 2,3 & 21 & & 10,5 & \\
\hline Kanada (1) & 2,1 & 2,5 & 19 & 10,1 & 9,4 & -7 \\
\hline Luxemburg & 2,2 & 2,8 & 27 & 7,4 & 11,9 & 61 \\
\hline Mexiko & 1,6 & 2,2 & 38 & 2,2 & 2,6 & 18 \\
\hline Neuseeland & 2,2 & 2,7 & 23 & & 10,1 & \\
\hline Niederlande (1) (2) & 2,4 & 3,1 & 29 & & & \\
\hline Norwegen & 3,4 & 4,2 & 24 & & 16,5 & \\
\hline Österreich & 3,9 & 4,9 & 26 & 7,2 & 7,8 & 8 \\
\hline Polen & 2,2 & 2,2 & 0 & 5,0 & 5,5 & 10 \\
\hline Schweden & 3,1 & 3,9 & 26 & & & \\
\hline Schweiz & & 3,9 & & 12,9 & 16,6 & 29 \\
\hline Slowakei (1) (2) & 3,4 & 3,4 & 0 & & & \\
\hline Slowenien & 2,2 & 2,5 & 14 & 6,9 & 8,2 & 19 \\
\hline Spanien & 3,2 & 3,8 & 19 & 3,6 & 5,2 & 44 \\
\hline Südkorea & 1,3 & 2,1 & 62 & 3,0 & 4,8 & 60 \\
\hline Tschechien & 3,4 & 3,7 & 9 & 7,6 & 8,1 & 7 \\
\hline Türkei (1) (2) & 1,3 & 1,7 & 31 & & & \\
\hline Ungarn & 2,7 & 3,1 & 15 & 5,3 & 6,3 & 19 \\
\hline USA (2) & 2,3 & 2,5 & 9 & & & \\
\hline Durchschnitt & 2,7 & 3,2 & 20 & 7,4 & 9,1 & 16,7 \\
\hline
\end{tabular}

(1) Ärzte: inkl. zusätzliche 5-10 Prozent nicht direkt an Patienten tätige Ärzte, z. B. Forscher, Ausbildner, Manager (im Gesundheitswesen)

(2) Pflegefachpersonen: inkl. zusätzliche 5-10 Prozent im Gesundheitswesen als Manager, Ausbildner, Forscher usw. tätige Pflegefachleute

Gesundheitswesens. Die Entwicklung der Personalbestände von 2000 bis 2012 gibt keinen Anlass zu Optimismus (Tab. 3). Die OECD-Ärztedichte hat in diesen 12 Jahren nur um 20 Prozent zugenommen, die Pflegendendichte um 22 Prozent. 2012 praktizierten in der Schweiz 3,9 Ärzte pro 1000 Einwohner (22 Prozent mehr als im OECD-Durchschnitt von 3,2 ). Die Schweiz und Schweden sind damit im 6. Rang von 30 Ländern.

Ganz anders ist die Lage bei den Pflegenden: In den 22 Ländern, für welche die OECD auch Zahlen für 2000 vorlegen kann, arbeiten im Schnitt 9,1 Pflegende pro 1000 Einwohner. Die Schweiz liegt mit nicht weniger als 16,6 Pflegenden an erster Stelle (82 Prozent mehr als in den 21 Vergleichsländern!). Dass man in unserem Land überall über Pflegepersonalmangel klagt und keineswegs über einen Dichtestress unter Pflegenden, weckt Zweifel an der OECDZahl 16,6 für die Schweiz. Tatsächlich liefert das Bundesamt für Statistik der OECD seit Jahren Zahlen zur Pflegendendichte, die nicht auf der gleichen Definition wie die der anderen Länder beruhen, obwohl das Obsan differenzierte Zahlen zur Verfügung stellt und daran ist, sie in Zusammenarbeit mit den verschiedenen Partnern noch weiter zu verfeinern. Der Schweizer Berufsverband der Pflegefachfrauen und Pflegefachmänner SBK protestiert seit längerem beim BAG und dem Bundesamt für Statistik gegen diese irreführende Praxis. Wenn die Schweiz weiterhin nicht kompatible Zahlen bereitstellen kann, wäre es besser, der OECD dazu überhaupt keine Zahl zu liefern. Informationen zur Pflege international geben eine neue Studie [12] sowie eine EU-Meinungsumfrage in allen Mitgliedsländern [13].

\section{Zufriedenheit und Qualität: Grossbritannien siegt, USA im Schlussrang}

«The Commonwealth Fund» ist eine private Stiftung in New York, die immer wieder interessante Studien publiziert. Tabelle 4 stammt aus Meinungsumfragen in 11 Ländern [14]. Nicht überraschend ist die miserable Bewertung des teuersten Gesundheitswesens der Welt: Die USA sind auf dem 11. und letzten Rang. 75 Prozent der befragten Amerikaner halten fundamentale Änderungen oder gar eine komplette Umgestaltung des Systems für notwendig. Dass aber in dieser Rangliste Grossbritannien mit deutlichem Vorsprung den ersten Platz erreicht, ist schon erstaunlich. Zwei Drittel der Briten finden, dass ihr kostengünstiges Gesundheitssystem gut funktioniert und nur kleine Änderungen nötig sind. Die Schweiz ist im zweiten Rang.

Der National Health Service NHS in Grossbritannien siegt nicht nur in dieser Meinungsumfrage, sondern auch in einer anderen Studie des Commonwealth Fund (15), welche eine Gesamtbeurteilung der Qualität des Gesundheitswesens vornimmt. Erfasst wurden 80 Leistungsindikatoren $\mathrm{zu}$ Themen wie Effektivität, Sicherheit, Koordination, Patientenzentrierung, Zugang zu Leistungen, Chancengleichheit, Gesundheitszustand. Die Studie beruht auf zahlreichen Untersuchungen der OECD, der WHO und des Commonwealth Fund. Die Qualitätsrangfolge: Grossbritannien, Schweiz, Schweden, Australien, Rang 5: Deutschland und Niederlande, Rang 7: Neuseeland und Norwegen, Frankreich, Kanada und als Schlusslicht im 11. Rang wiederum die USA.

Die Studie des Commonwealth Fund stösst vor allem in den USA auf grosse Beachtung, aber auch auf Kritik [16]. Sie illustriert, dass die Qualität eines 
Tabelle 4

Zufriedenheit mit dem Gesundheitssystem.

A: Funktioniert gut, nur kleine Änderungen nötig;

B: Fundamentale Änderungen nötig;

C: Komplette Umgestaltung nötig. (Zahlen in Prozent).

\begin{tabular}{lllr} 
& A & B & C \\
Grossbritannien & 63 & 33 & 4 \\
\hline Schweiz & 54 & 40 & 6 \\
\hline Niederlande & 51 & 44 & 5 \\
\hline Australien & 48 & 43 & 9 \\
Neuseeland & 47 & 45 & 8 \\
Norwegen & 46 & 42 & 12 \\
\hline Schweden & 44 & 46 & 10 \\
\hline Deutschland & 42 & 48 & 10 \\
Kanada & 42 & 50 & 8 \\
Frankreich & 40 & 49 & 11 \\
\hline USA & 25 & 48 & 27 \\
Durchschnitt & 46 & 44 & 10
\end{tabular}

Gesundheitssystems schwierig zu definieren und zu messen ist. Internationale Vergleiche mit einheitlicher Methodik sind aber lehrreich, weil die Gesundheitspolitik in allen Ländern zu stark national ausgerichtet ist und von Erfahrungen im Ausland viel lernen könnte und sollte. Was wäre die Medizin, wenn auch sie einen so engen Horizont hätte?

\section{Literatur}

1 Bundesamt für Statistik, Kosten und Finanzierung des Gesundheitswesens, 2012 (provisorisch). www.bfs. admin.ch/bfs/portal/de/index/themen/14/05.html

2 Für weitere internationale Vergleiche s. www. kingsfund.org.uk/sites/files/kf/field/field_pdf/ Library-reading-list-international-health-carecomparisons-Jan2013.pdf

3 Frequently requested data, kostenlos abrufbar unter www.oecd.org/health/health-systems/oecd-healthstatistics-2014-frequently-requested-data.htm

4 Details im OECD-Katalog: http://issuu.com/oecd. publishing/docs/oecd_subscriptions-catalog_2014

5 www.oecd.org/els/health-systems/List-of-variablesOECD-Health-Statistics-2014.pdf

6 Deprimierend sind Ausgabenvergleiche mit den ärmsten Staaten der Welt, die alle nicht OECD-Mitglieder sind. In der Demokratischen Republik Kongo und in Eritrea beliefen sich 2012 die jährlichen Pro-KopfAusgaben für die Gesundheit auf ganze 15 USD - 583-mal weniger als in den USA (Weltbank, Health expenditure per capita, 2014, http://data.worldbank org/indicator/SH.XPD.PCAP).

7 Kocher G. Gesundheit und Gesundheitssysteme in Europa - ein aktueller Ländervergleich. Schweiz Ärztezeitung. 2013(9):345-7.

8 Kocher G, Rentchnick P, Verlag Hans Huber, Bern (1980) und Editions Pierre-Marcel Favre, Lausanne (Chère médecine, 1979). Die in diesem Abschnitt zitierten Berechnungen stammen aus diesem Buch.

9 Smith $\mathrm{M}$ et al. Best Care at Lower Cost, The Path to Continuously Learning Health Care in America. Washington: The National Academies Press; 2012.

10 BAG. Bern. www.gesundheit2020.ch

11 S. dazu: Frey M et al. Direkte Gesundheitsausgaben der privaten Haushalte. Out-Of-Pocket-Zahlungen und Kostenbeteiligungen in der Schweiz und im internationalen Vergleich. Obsan; 2011. www.obsan.admin.ch/ bfs/obsan/de/index/05/publikationsdatenbank. html?publicationID $=4523$

12 Schwendimann et al. Das Pflegefachpersonal in Schweizer Spitälern im europäischen Vergleich. Obsan; 2014. www.obsan.admin.ch/bfs/obsan/de/ index/05/publikationsdatenbank. html?publicationID=5607

13 Patient Safety and Quality of Care, Special Eurobarometer 411. European Commission. Brussels; 2014. http://ec.europa.eu/health/patient_safety/docs/ ebs_411_en.pdf

14 The Commonwealth Fund 2013 International Health Policy Survey in Eleven Countries. New York; 2013. www.commonwealthfund.org/publications/ surveys/2013/2013-commonwealth-fundinternational-health-policy-survey

15 Davis K et al. Mirror, Mirror on the Wall. How the Performance of the U.S. Health Care System Compares Internationally, 2014 Update. The Commonwealth Fund. New York; June 2014 www.commonwealthfund.org/publications/ fund-reports/2014/jun/mirror-mirror

16 Beispiel: Gur-Arie M. Digging deeper into the Commonwealth Fund health rankings. www.kevinmd.com/blog/2014/07/diggingdeeper-commonwealth-fund-health-rankings.html

\section{Articles interactifs}

Vous souhaitez commenter cet article? II vous suffit pour cela d'utiliser la fonction «Ajouter un commentaire» dans la version en ligne. Vous pouvez également consulter les remarques de vos confrères sous: www.bullmed.ch/ numero-actuel/articles-interactifs/ 